UP-HEP-9702

hep-ph/9702321

February 12, 1997

\title{
Gauge Dependence of Lower Bounds on the Higgs Mass Derived from Electroweak Vacuum Stability Constraints
}

\author{
Will Loinaz由 \\ R.S. Willey ${ }^{2}$ \\ Department of Physics and Astronomy \\ University of Pittsburgh, Pittsburgh, PA 15260, USA
}

\begin{abstract}
We examine the gauge dependence of lower bounds on the Higgs mass obtained from the requirement that the electroweak vacuum be the global minimum of the effective potential. We study a simple model, the spontaneouslybroken Abelian Higgs model coupled to a chiral quark doublet in two-parameter $R_{\xi, u}$ gauge and demonstrate that the lower bounds on the Higgs mass obtained in this model are dependent on the choice of gauge parameters. We discuss the significance of this result for calculations in the Standard Model.
\end{abstract}

\footnotetext{
${ }^{1}$ E-mail: loinaz+@pitt.edu

${ }^{2}$ E-mail: willey@vms.cis.pitt.edu
} 


\section{Introduction}

In the absence of direct observation of the Standard Model Higgs boson, considerable effort has been expended on the study of its properties through indirect means. There has been a series of papers deriving the lower bound on the Higgs mass, based on considerations of the stability of the electroweak vacuum (see [i] for a comprehensive review of work through 1990). In the strongest form of the vacuum stability bound, it is assumed that the electroweak vacuum is the absolute minimum of the effective potential $\left(V_{\text {eff }}[\Phi]\right)$, at least up to some 'new physics' scale at which the effects of unknown high-scale physics become significant and the low-energy model is no longer appropriate. This places restrictions on the running quartic coupling $\lambda$ at the high scale. After running $\lambda$ down to the electroweak scale, this is converted into a restriction on the Higgs pole mass.

In this paper we investigate the consequences of the gauge dependence of the effective potential for these calculations. The gauge dependence of the effective potential was pointed out in the early 70s [2]. The effective potential is the sum of one-particle irreducible (1-PI) Greens functions at zero external momentum. In a gauge theory with massive scalars these are offshell quantities, and thus in general gauge-dependent. It is that known the value of $V_{\text {eff }}[\Phi]$ at any of its extrema, $\Phi_{i}$, is gauge independent. However, the locations of these extrema along the $\Phi$ axis, the $\Phi_{i}$ themselves, are gauge-dependent. In practice, the input condition has been that the renormalization group (RG) improved perturbative $V_{\text {eff }}(\Phi)$ should not fall through zero for variable $\Phi$ less than some chosen maximum ('cutoff') value $\Phi_{\max }$. (In some studies, the t'Hooft dimensional regularization scale $\kappa$ is identified with the variable $\Phi$. Then it is $\left.\kappa_{\max }\right)$. But the condition $V_{\text {eff }}\left(\Phi_{\max }\right) \geq 0$ is manifestly gauge dependent (the arbitrarily chosen $\Phi_{\max }$ is not a stationary point, so the value of $V_{\text {eff }}$ at $\Phi_{\max }$ depends on the gauge ). Alternatively, one could require that the derivative $\frac{d V_{\mathrm{eff}}[\Phi]}{d \Phi}$ not go to zero before $\Phi_{\max }$, since $\left[\frac{d V_{\text {eff }}[\Phi]}{d \Phi}\right]_{\Phi_{0}}=0$ identifies $\Phi_{0}$ as a stationary point. But the solution $\Phi_{0}$ is also gauge dependent (it is the vev of a quantum field which is not invariant under the transformations which have been gauged, e.g. $O(N)$ ). Thus the proposed condition $\Phi_{0}$ greater than or equal to some arbitrarily fixed $\Phi_{\text {max }}$ is also gauge dependent. The relation between the Higgs mass and the scale of new physics, defined either as a value of $\Phi$ at the extremum of the effective potential or a value of $\Phi$ at which the effective potential achieves some fixed value, relates a physical quantity to an unphysical quantity. It thus cannot be expected to be gauge invariant.

The tree approximation to $V_{\text {eff }}$ is gauge invariant. If one restricts the calculation to the replacement of the fixed quartic scalar coupling by its $\mathrm{RG}$ running version in the tree approximation for $V_{\text {eff }}$ and uses a gauge invariant definition of the $\overline{M S}$ running masses and gauge invariant $\beta$ functions, the calculation never encounters any gauge dependence (i.e. the gauge parameter $\xi$ never appears). However, if one attempts to improve the estimate by including some information from the one-loop contributions to $V_{\text {eff }}$, the results are essentially infected by the gauge dependence described above. We conclude that the original estimate cannot be improved. No model-independent error estimate is possible in the context of a calculation based on the effective potential. The reader who is convinced by these simple considerations may skip the lengthy calculations that follow and skip to section 5 where we discuss some possible formulations not involving the effective potential which are free of problems with gauge dependence.

In this paper we demonstrate the gauge-dependence of the lower mass bound of the Higgs by explicit calculation in a toy model. Our model consists of a spontaneouslybroken Abelian Higgs theory coupled to a doublet of chiral fermions ('top' and 'bot- 
tom'), one of which obtains a mass through the spontaneous symmetry breaking. For certain regions of the parameter space this model will display the qualitative features of the Standard Model necessary to study to electroweak vacuum stability. In section 2 we outline write out the Lagrangian for the model in a two- parameter gauge, the $R_{\xi, u}$ gauges. We outline the one-loop $\overline{M S}$ renormalization of the theory for $\mu^{2}>0$ and $\mu^{2}<0$ and give an explicit expression for the vev of the Higgs field. In section 3 we calculate the one-loop effective potential in general $R_{\xi, u}$ gauges and check that calculating the location of the extremum of this effective potential gives the same vev as the perturbation theory calculation in the broken symmetry phase. We then do the RG improvement to sum the possibly large logarithms introduced by the one-loop contribution to the effective potential. In section 4 we discuss the connection between the RG-improved effective potential and the electroweak vacuum instability scale. We show explicitly that electroweak vacuum instability scales defined by the location of some feature of the effective potential along the $\Phi$ axis are gauge-dependent and will pass this gauge dependence into a mass bound on the Higgs. In section 5 we discuss possible alternative methods for deriving lower bounds on the Higgs mass, and in section 6 we summarize our conclusions.

\section{The Model}

\subsection{Lagrangian}

The model which we study is the spontaneously-broken Abelian Higgs model coupled to a chiral fermion doublet. Over some region of its parameter space this model displays the same sort of vacuum instability due to heavy fermion loops as arises in the Standard Model. We stress that we do not propose that a numerical lower bound on the Standard Model Higgs boson be calculated from this model. It is merely a toy model for illustrating certain issues of principle which we wish to present without the unnecessary complications of a nonabelian gauge theory. Note in particular that the fact that a $U(1)$ gauge theory is not asymptotically free poses no difficulty as long as vacuum instability scales are well below the Landau singularities of the couplings.

The Lagrangian is

$$
\begin{gathered}
\mathcal{L}=\mathcal{L}_{c l}^{A H}+\mathcal{L}_{c l}^{f}+\mathcal{L}_{g f}+\mathcal{L}_{F P} \\
\mathcal{L}_{c l}^{A H}=-\frac{1}{4}\left(\partial_{\mu} B_{0 \nu}-\partial_{\nu} B_{0 \mu}\right)^{2}+\frac{1}{2}\left(\partial_{\mu} h_{0}\right)^{2}+\frac{1}{2}\left(\partial_{\mu} \chi_{0}\right)^{2} \\
+g_{0}\left[\left(\partial_{\mu} h_{0}\right) \chi_{0}-\left(\partial_{\mu} \chi_{0}\right) h_{0}\right] B_{0}^{\mu}+\frac{1}{2} g_{0}^{2} B_{0 \mu} B_{0}^{\mu}\left(h_{0}^{2}+\chi_{0}^{2}\right) \\
-\frac{1}{2} \mu_{0}^{2}\left(h_{0}^{2}+\chi_{0}^{2}\right)-\frac{\lambda_{0}}{4}\left(h_{0}^{2}+\chi_{0}^{2}\right)^{2} \\
\mathcal{L}_{c l}^{f}=i \bar{t}_{0 L} \not t_{0 L}+i \bar{t}_{0 R} \not t_{0 R}+i \bar{b}_{0 L} \not \partial b_{0 L}+i \bar{b}_{0 R} \not \partial b_{0 R}+i g_{0}\left[\bar{t}_{0 L} \not B_{0} b_{0 L}-\bar{b}_{0 L} \not B_{0} t_{0 L}\right] \\
-y_{t 0}\left[\bar{t}_{0 L} t_{0 R}+\bar{t}_{0 R} t_{0 L}\right] h_{0}-y_{t 0}\left[\bar{b}_{0 L} t_{0 R}+\bar{t}_{0 R} b_{0 L}\right] \chi_{0}
\end{gathered}
$$

As usual, $(h, \chi)$ is a doublet of real scalar fields. $t$ and $b$ are fermion fields. Their left-handed projections $\left(t_{L}, b_{L}\right)$ form a doublet under the local gauge transformation, while their right-handed projections transform as a singlet. Spontaneous symmetry 
breaking along the $h$ direction then gives a tree-level $(\overline{M S})$ mass to the Higgs scalar, the vector boson, and the $t$ fermion. The Goldstone boson $\chi$ receives in the brokensymmetry phase no tree-level mass from $\mathcal{L}_{c l}$, nor does $b$.

To study the gauge-dependence of the mass bound that results from this model, we choose to work in a two-parameter gauge, the $R_{\xi, u}$ gauge. The gauge-fixing term is:

$$
\mathcal{L}_{g f}=-\frac{1}{2 \xi_{0}}\left(\partial_{\mu} B_{0}^{\mu}+\xi_{0} u_{0} g_{0} \chi_{0}\right)^{2}
$$

and the corresponding Fadeev-Popov ghost term is:

$$
\mathcal{L}_{F P}=-\bar{c}_{0} \not c_{0}-\xi_{0} u_{0} g_{0}^{2} \bar{c}_{0} c_{0} h_{0}
$$

Special cases of this gauge include the Landau gauges $(\xi=0)$, 't Hooft $R_{\xi}$ gauges [3] $(u=v$, the tree-level Higgs vev) and Fermi gauges $(u=0)$.

The gauge-fixing term is chosen anticipating that the spontaneous symmetry breaking will be in the $h$ direction. In the $R_{\xi}$ gauges, the gauge-fixing term cancels the tree level $\partial B-\chi$ mixing term generated in the renormalized classical Lagrangian by the shift of the $h$ field. This is especially convenient for perturbative calculations in the broken-symmetry phase. The gauge-fixing term explicitly breaks the $O(2)$ symmetry of $\mathcal{L}_{c l}^{A H}$, giving the $\chi$ a tree-level mass. The ghost also receives a tree-level mass under SSB. Except in the Fermi and Landau gauges, the ghost is not free.

\section{$2.2 \overline{M S}$ Renormalization}

We renormalize the theory by rescaling the parameters and fields of the Lagrangian by multiplicative renormalization factors $Z$ :

$$
\begin{array}{rll}
g_{0}=Z_{g} g & \lambda_{0}=Z_{\lambda} \lambda & y_{t 0}=Z_{y} y_{t} \\
\mu_{0}^{2}=Z_{\mu^{2}} \mu^{2} & \xi_{0}=Z_{\xi} \xi & u_{0}=Z_{u} u
\end{array}
$$

Among the fields, members of an $O(2)$ doublet receive a common renormalization.

$$
\begin{aligned}
& B_{0}^{\mu}=Z_{B}^{\frac{1}{2}} B^{\mu} \quad\left(h_{0}, \chi_{0}\right)=Z_{\phi}^{\frac{1}{2}}(h, \chi) \quad\left(t_{L 0}, b_{L 0}\right)=Z_{L}^{\frac{1}{2}}\left(t_{L}, b_{L}\right) \\
& t_{R 0}=Z_{t}^{\frac{1}{2}} t_{R} \quad b_{R 0}=Z_{b}^{\frac{1}{2}} b_{R} \quad c_{0}=Z_{c}^{\frac{1}{2}} c
\end{aligned}
$$

Writing

$$
Z=1+(Z-1)=1+\delta Z
$$

and substituting (6), (7), and (8) into the Lagrangian generates the counterterms for the theory. Various Ward/BRST identities determine that no counterterms are generated by the renormalization transformations of the gauge-fixing and ghost Lagrangians.

The definition of the counterterms and the physical meaning of the renormalized parameters depends on the renormalization scheme. Here we work in $\overline{M S}$. The counterterms may then be calculated in either the symmetric or broken symmetry phase of the theory [4].

The simplest case, $u=0$ gauges in the symmetric phase $\left(\mu^{2}>0\right)$ is (aside from the fermions) simply scalar QED (in $O(2)$ rather than $U(1)$ variables). The $\delta Z \mathrm{~s}$ 


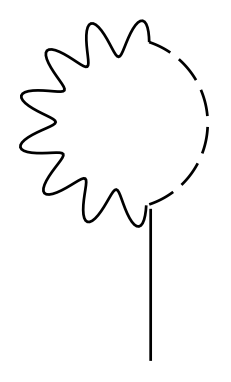

Figure 1: Divergent Higgs One-Point Function for $\mu^{2}>0$ in $R_{\xi, u}$ Gauge

are calculated by cancelling the divergences of the two and four point 1-PI functions $\left(\Gamma_{(n)}\right)$. The relations between $Z_{g}, Z_{B}$ and $Z_{\xi}$ are familiar from QED Ward identities. A particularly useful feature arises from the relation $Z_{\xi} Z_{g}^{2}=1$. This implies that $\xi g^{2}$ is a renormalization group invariant, although individually the two factors run. Thus, once $\xi g^{2}$ is fixed to some initial value it remains unchanged under RG running. Since $\xi$ appears only in the combination $\xi g^{2}$ in the effective potential, we may simply set the value of $\xi g^{2}$ and never consider the running of $\xi$ alone.

In the more general $R_{\xi, u}$ gauge complications arise as a result of the new pieces of the gauge-fixing term which explicitly break the global $O(2)$ symmetry of the $\mathcal{L}_{c l}$. In particular, the tree-level $\partial B-\chi$ mixing induced by this gauge-fixing gives rise to a divergent Higgs one-point function (see Fig. 1). Thus the Higgs field acquires a divergent one-loop vev even for $\mu^{2}>0$. To perturb about the correct vacuum, we make a shift of the field

$$
h=h^{\prime}+\delta w
$$

and choose $\delta w$ such that

$$
\left\langle 0\left|h^{\prime}\right| 0\right\rangle=0 .
$$

Explicitly to one-loop order we find

$$
\delta w=-\frac{\xi g^{2} u}{(4 \pi)^{2}}\left[-\Delta_{\epsilon}+\log \frac{\mu^{2}}{\kappa^{2}}-1\right]
$$

where

$$
\Delta_{\epsilon}=\frac{2}{4-d}-\gamma_{E}+\log 4 \pi
$$

is the standard $\overline{M S} \mathrm{UV}$ subtraction in $d$ dimensions and $\kappa$ is the renormalization scale. This shift generates one-loop counterterms which in addition to eliminating the one-point function of the shifted field $h^{\prime}$ also renders finite the bosonic threepoint functions (which also arise as a result of $\partial B-\chi$ mixing). For $\mu^{2}>0, \delta w \stackrel{u \rightarrow 0}{\longrightarrow} 0$ and we return smoothly to the limit of the usual symmetric phase described above.

Perturbation theory for $\mu^{2}<0$ is most straightforward in the 't Hooft $R_{\xi}$ gauges $(u=v)$. For $\mu^{2}<0$ the symmetry is spontaneously broken already at the tree level. To perturb about the correct vacuum we shift the field $h$ by the exact vev $V$. The exact vev is determined as a function of the parameters of the theory order-by-order in perturbation theory by the requirement that the one-point function of the shifted field $\hat{\Gamma}_{(1)}$ vanish order-by-order in perturbation theory.

$$
h=\hat{h}+V \Longrightarrow\langle 0|\hat{h}| 0\rangle=0
$$




$$
V=v+\delta V
$$

The gauge-fixing term with $u=v$ cancels the tree-level $\partial B-\chi$ term generated in $\mathcal{L}_{c l}$ by the field shift, leaving $\delta V$ to act as a one-loop counterterm. The vev $V$ computed to one-loop order is

$$
\begin{aligned}
V= & v+\frac{v}{(4 \pi)^{2}}\left[\xi g^{2} \Delta_{\epsilon}-3 \lambda\left(\log \frac{m_{h}^{2}}{\kappa^{2}}-1\right)-\frac{1}{2} \frac{g^{4}}{\lambda}\left(3 \log \frac{M^{2}}{\kappa^{2}}-1\right)\right. \\
& \left.-\frac{1}{2} \xi g^{2}\left(\log \frac{\xi M^{2}}{\kappa^{2}}-1\right)+2 N_{f} \frac{y_{t}^{4}}{\lambda}\left(\log \frac{m_{t}^{2}}{\kappa^{2}}-1\right)\right]
\end{aligned}
$$

In terms of the renormalized parameters of the theory, $\mu^{2}(<0), \lambda, g^{2}$, and $y_{t}^{2}$ the masses and tree-level vev in (15) are

$$
\begin{aligned}
v & =\sqrt{\frac{-\mu^{2}}{\lambda}} \\
m_{h}^{2} & =-2 \mu^{2} \\
M & =g v \\
m_{t} & =y_{t} v
\end{aligned}
$$

Note that $\delta V$ is explicitly gauge-dependent and contains and a UV pole $\left(\Delta_{\epsilon}\right)$ [4, 5, 6]. The subtractions implied by $\delta V$ and the $\delta Z$ s eliminate $\hat{\Gamma}_{(1)}$ and render all the other $\hat{\Gamma}_{(n)}$ finite.

For general $u \neq v$ there is again the complication of uncancelled tree- level $\partial B-\chi$ mixing, but the procedure is the same. Again requiring $\left\langle 0\left|h^{\prime}\right| 0\right\rangle=0$ we calculate the corrections to the vev

$$
\begin{aligned}
\delta V & =-\frac{\xi g^{2} u}{(4 \pi)^{2}}\left[-\Delta_{\epsilon}+\log \frac{\xi g^{2} u v}{\kappa^{2}}-\frac{3}{2}\right]-\frac{v}{(4 \pi)^{2}}\left[3 \lambda\left(\log \frac{2 \lambda v^{2}}{\kappa^{2}}-1\right)\right. \\
& \left.+\frac{1}{2} \frac{g^{4}}{\lambda}\left(3 \log \frac{g^{2} v^{2}}{\kappa^{2}}-1\right)-\frac{1}{2} \xi g^{2}\left(\log \frac{\xi g^{2} u v}{\kappa^{2}}-2\right)-2 N_{f} \frac{y_{t}^{4}}{\lambda}\left(\log \frac{m_{t}^{2}}{\kappa^{2}}-1\right)\right]
\end{aligned}
$$

Note that for $u=v$ this reproduces the $R_{\xi}$ gauge result (15). Difficulties arise in taking the $u \rightarrow 0$ (Fermi gauge) limit. IR divergences resulting from the massless Goldstone boson spoil the calculation of the one-loop correction to the vev. Fermi gauges are in any case not especially well-suited to broken-symmetry phase calculations, since the $\partial B-\chi$ mixing from $\mathcal{L}_{c l}$ is not cancelled by the gauge-fixing term. $R_{\xi, u}$ gauges in general also contains $\partial B-\chi$ mixing, but for the gauge $u=v\left(R_{\xi}\right.$ gauges) the tree-level $\partial B-\chi$ mixing term is cancelled. Thus, Fermi gauge is clearly a natural choice for symmetric phase perturbative calculations, as $R_{\xi}$ gauges are for broken-symmetry phase calculation.

\section{The Renormalization-Group Improved Effective Potential}




\subsection{One-loop Effective Potential}

The quantity we will refer to as the effective potential, $V_{\text {eff }}[\phi]$, is the sum of 1PI diagrams with any number of external Higgs legs, each carrying zero external momentum.

$$
V_{\text {eff }}(\Phi)=-\sum_{n=1}^{\infty} \frac{1}{n !} \Phi^{n} \Gamma_{(n)}\left(p_{i}=0\right)
$$

This is in fact not the effective potential, since it is not convex and may be complex. We sidestep entirely the discussion of convexity, referring the reader to the literature (e.g. [7], Appendix of [1]) for the argument that this is indeed the appropriate function to study. As mentioned previously, in a theory with massive scalars the Greens functions at zero external momentum are in general gauge-dependent, and as a result so is $V_{\text {eff }}[\phi]$.

The calculation of the one-loop effective potential is most simply carried out via the tadpole method [8]. The main complication is that one has to deal with mixed $\partial B-\chi$ propagators in any but Landau gauge. The full one-loop expression is:

$$
\begin{aligned}
V_{\text {eff }}[\Phi]= & \frac{1}{2} \mu^{2} \Phi^{2}+\frac{\lambda}{4} \Phi^{4}+\frac{1}{4} \frac{H^{4}[\Phi]}{(4 \pi)^{2}}\left[\log \frac{H^{2}[\Phi]}{\kappa^{2}}-\frac{3}{2}\right] \\
& +\frac{3}{4} \frac{B^{4}[\Phi]}{(4 \pi)^{2}}\left[\log \frac{B^{2}[\Phi]}{\kappa^{2}}-\frac{5}{6}\right]-\frac{2}{4} \frac{G^{4}[\Phi]}{(4 \pi)^{2}}\left[\log \frac{G^{2}[\Phi]}{\kappa^{2}}-\frac{3}{2}\right] \\
& +\frac{1}{4} \frac{k_{+}^{4}[\Phi]}{(4 \pi)^{2}}\left[\log \frac{k_{+}^{2}[\Phi]}{\kappa^{2}}-\frac{3}{2}\right]+\frac{1}{4} \frac{k_{-}^{4}[\Phi]}{(4 \pi)^{2}}\left[\log \frac{k_{-}^{2}[\Phi]}{\kappa^{2}}-\frac{3}{2}\right] \\
& -N_{f} \frac{Y^{4}[\Phi]}{(4 \pi)^{2}}\left[\log \frac{Y^{2}[\Phi]}{\kappa^{2}}-\frac{3}{2}\right]+\xi g^{2} u \Phi\left(\mu^{2}+\lambda \Phi^{2}\right)\left[\frac{-\Delta_{\epsilon}}{(4 \pi)^{2}}\right]
\end{aligned}
$$

where

$$
\begin{aligned}
H^{2}[\Phi]= & \mu^{2}+3 \lambda \Phi^{2} \\
B^{2}[\Phi]= & g^{2} \Phi^{2} \\
G^{2}[\Phi]= & \xi g^{2} u \Phi \\
k_{ \pm}^{2}[\Phi]= & \frac{1}{2}\left[\mu^{2}+\lambda \Phi^{2}+2 \xi g^{2} u \Phi\right] \pm \\
& \frac{1}{2} \sqrt{\left(\mu^{2}+\lambda \Phi^{2}\right)\left(\mu^{2}+\lambda \Phi^{2}+4 \xi g^{2} \Phi(u-\Phi)\right)} \\
Y^{2}[\Phi]= & y_{t}^{2} \Phi^{2}
\end{aligned}
$$

$H, B, Y$ and $G$ denote contributions from Higgs, vector boson, heavy fermion and Fadeev-Popov ghost loops, respectively. The $k_{ \pm}^{2}$ terms arise from the $\partial B-\chi$ sector. This result agrees with that obtained in [9], with the exception of a difference in the non-log piece of the B term 1 and the presence of the UV pole. It is manifestly gauge-dependent, and the pole term exists for $\xi, u \neq 0$.

\footnotetext{
${ }^{1}$ We suspect that this is due to a neglected $\frac{d-4}{\epsilon}=-1$ contribution in the calculation of the gauge boson piece.
} 
The expression for the $V_{\text {eff }}$ can be checked by comparing the expressions for the extrema to those obtained by direct perturbative calculation. The extrema of $V_{\text {eff }}$ are found by solving

$$
\left[\frac{\partial V_{\mathrm{eff}}[\Phi]}{\partial \Phi}\right]_{\langle\Phi\rangle}=0
$$

Writing the extrema of $V_{\text {eff }}$ in a loop expansion as

$$
\langle\Phi\rangle=\left\langle\Phi_{0}\right\rangle+\left\langle\Phi_{1}\right\rangle+\ldots
$$

this becomes

$$
\begin{aligned}
0 \stackrel{1-\text { loop }}{=}\left[\frac{\partial V_{\mathrm{eff}}^{0}[\Phi]}{\partial \Phi}\right]_{\left\langle\Phi_{0}\right\rangle+\left\langle\Phi_{1}\right\rangle}+\left[\frac{\partial V_{\mathrm{eff}}^{1}[\Phi]}{\partial \Phi}\right]_{\left\langle\Phi_{0}\right\rangle} \\
=\left[\frac{\partial V_{\mathrm{eff}}^{0}[\Phi]}{\partial \Phi}\right]_{\left\langle\Phi_{0}\right\rangle}+\left[\frac{\partial^{2} V_{\mathrm{eff}}^{0}[\Phi]}{\partial \Phi^{2}}\right]_{\left\langle\Phi_{0}\right\rangle}\left\langle\Phi_{1}\right\rangle+\left[\frac{\partial V_{\mathrm{eff}}^{1}[\Phi]}{\partial \Phi}\right]_{\left\langle\Phi_{0}\right\rangle} .
\end{aligned}
$$

Solving for the $\left\langle\Phi_{0}\right\rangle$ and $\left\langle\Phi_{1}\right\rangle$ gives

$$
\begin{aligned}
\left\langle\Phi_{0}\right\rangle= & \sqrt{\frac{-\mu^{2}}{\lambda}} \equiv v \\
\left\langle\Phi_{1}\right\rangle= & -\left[\frac{\partial^{2} V_{\mathrm{eff}}^{0}[\Phi]}{\partial \Phi^{2}}\right]_{\left\langle\Phi_{0}\right\rangle}^{-1}\left[\frac{\partial V_{\mathrm{eff}}^{1}[\Phi]}{\partial \Phi}\right]_{\left\langle\Phi_{0}\right\rangle}=-\frac{1}{2 \lambda v^{2}}\left[\frac{\partial V_{\mathrm{eff}}^{1}[\Phi]}{\partial \Phi}\right]_{\left\langle\Phi_{0}\right\rangle} \\
= & -\frac{\xi g^{2} u}{(4 \pi)^{2}}\left[-\Delta_{\epsilon}+\log \frac{\xi g^{2} u v}{\kappa^{2}}-\frac{3}{2}\right] \\
& -\frac{v}{(4 \pi)^{2}}\left[3 \lambda\left(\log \frac{2 \lambda v^{2}}{\kappa^{2}}-1\right)+\frac{1}{2} \frac{g^{4}}{\lambda}\left(3 \log \frac{g^{2} v^{2}}{\kappa^{2}}-1\right)\right. \\
& \left.-\frac{1}{2} \xi g^{2}\left(\log \frac{\xi g^{2} u v}{\kappa^{2}}-2\right)-2 N_{f} \frac{y_{t}^{4}}{\lambda}\left(\log \frac{m_{t}^{2}}{\kappa^{2}}-1\right)\right]
\end{aligned}
$$

which agrees with the perturbative result. We can also see that the value of $V_{\text {eff }}$ at the extremum is gauge-invariant:

$$
\begin{aligned}
V_{\text {eff }}[\langle\Phi\rangle] \stackrel{1-\text { loop }}{=} & V_{\text {eff }}^{0}\left[\left\langle\Phi_{0}\right\rangle+\left\langle\Phi_{1}\right\rangle\right]+V_{\text {eff }}^{1}\left[\left\langle\Phi_{0}\right\rangle\right] \\
= & V_{\text {eff }}^{0}\left[\left\langle\Phi_{0}\right\rangle\right]+\left[\frac{\partial V_{\text {eff }}^{0}[\Phi]}{\partial \Phi}\right]_{\left\langle\Phi_{0}\right\rangle}\left\langle\Phi_{1}\right\rangle+V_{\text {eff }}^{1}\left[\left\langle\Phi_{0}\right\rangle\right] \\
= & V_{\text {eff }}^{0}\left[\left\langle\Phi_{0}\right\rangle\right]+V_{\text {eff }}^{1}\left[\left\langle\Phi_{0}\right\rangle\right] \\
= & -\frac{1}{4} \lambda v^{4}+\frac{1}{4} \frac{m_{h}^{4}}{(4 \pi)^{2}}\left[\log \frac{m_{h}^{2}}{\kappa^{2}}-\frac{3}{2}\right] \\
& +\frac{3}{4} \frac{m_{B}^{4}}{(4 \pi)^{2}}\left[\log \frac{m_{B}^{2}}{\kappa^{2}}-\frac{5}{6}\right]-N_{f} \frac{m_{t}^{4}}{(4 \pi)^{2}}\left[\log \frac{m_{t}^{2}}{\kappa^{2}}-\frac{3}{2}\right]
\end{aligned}
$$


Upon substituting $\Phi=\left\langle\Phi_{0}\right\rangle$ into (22) the UV pole disappears, the Fadeev-Popov ghost term cancels the $k_{ \pm}^{2}$ terms, and the resulting expression (33) is gauge-independent, as expected.

Observe that in the large-field limit $\Phi \gg u, \mu V_{\text {eff }}$ simplifies to

$$
V_{\text {eff }}[\Phi] \approx \frac{1}{4} \Phi^{4} \lambda_{\text {eff }}[\Phi]
$$

where

$$
\begin{aligned}
\lambda_{\text {eff }}[\Phi] \equiv & \lambda+\Delta \lambda[\Phi] \\
\Delta \lambda \equiv & \frac{9 \lambda^{2}}{(4 \pi)^{2}}\left[\log \frac{3 \lambda \Phi^{2}}{\kappa^{2}}-\frac{3}{2}\right]+\frac{3 g^{4}}{(4 \pi)^{2}}\left[\log \frac{g^{2} \Phi^{2}}{\kappa^{2}}-\frac{5}{6}\right] \\
& -N_{f} \frac{4 y_{t}^{4}}{(4 \pi)^{2}}\left[\log \frac{y_{t}^{2} \Phi^{2}}{\kappa^{2}}-\frac{3}{2}\right]+\frac{\tilde{k}_{+}^{4}}{(4 \pi)^{2}}\left[\log \frac{\tilde{k}_{+}^{2} \Phi^{2}}{\kappa^{2}}-\frac{3}{2}\right] \\
& +\frac{\tilde{k}_{-}^{4}}{(4 \pi)^{2}}\left[\log \frac{\tilde{k}_{-}^{2} \Phi^{2}}{\kappa^{2}}-\frac{3}{2}\right]
\end{aligned}
$$

and

$$
\tilde{k}_{ \pm}^{2}=\frac{1}{2}\left\{\lambda \pm \sqrt{\lambda\left(\lambda-4 \xi g^{2}\right)}\right\}
$$

Since $u$ is a gauge parameter, we are free to choose it as large or small as we wish. We use this freedom to assume $\Phi \gg u$, neglect $u$ and focus on the $\xi$ dependence of the quantities of interest. Since the $u$-dependence has been dropped, this is just what we would get from a calculation in Fermi gauge (again also neglecting $\mu^{2}$ ). Since $k_{ \pm}^{2}$ are complex conjugates (as are $\tilde{k}_{ \pm}^{2}$ ), their combined contribution is real regardless the sign of $\lambda$ or $\lambda-4 \xi g^{2}$. However, the contribution from the Higgs loop becomes complex for $\lambda<0$ if the running $\lambda$ is used inside the one-loop effective potential, as would be the case in [10, 11, 12] had the Higgs loop term been retained.

Taking the limit $\frac{\xi g^{2}}{\lambda} \ll 1, u \ll \Phi$ elucidates the structure of the gauge-dependent pieces. In this limit,

$$
\begin{aligned}
G^{2}[\Phi] & \sim 0 \\
k_{+}^{2}[\Phi] & \sim \lambda \Phi^{2}\left[1-\frac{\xi g^{2}}{\lambda}\right]-\frac{\left(\xi g^{2}\right)^{2}}{\lambda} \Phi^{2}+\mathcal{O}\left(\left(\xi g^{2}\right)^{3}\right) \\
k_{-}^{2}[\Phi] & \sim \xi g^{2} \Phi^{2}+\frac{\left(\xi g^{2}\right)^{2}}{\lambda} \Phi^{2}+\mathcal{O}\left(\left(\xi g^{2}\right)^{3}\right)
\end{aligned}
$$

and

$$
\begin{aligned}
\lambda_{\text {eff }}[\Phi] \equiv & \lambda+\Delta \lambda \\
\Delta \lambda \equiv & \frac{9 \lambda^{2}}{(4 \pi)^{2}}\left[\log \frac{3 \lambda \Phi^{2}}{\kappa^{2}}-\frac{3}{2}\right]+\frac{3 g^{4}}{(4 \pi)^{2}}\left[\log \frac{g^{2} \Phi^{2}}{\kappa^{2}}-\frac{5}{6}\right] \\
& -N_{f} \frac{4 y_{t}^{4}}{(4 \pi)^{2}}\left[\log \frac{y_{t}^{2} \Phi^{2}}{\kappa^{2}}-\frac{3}{2}\right]+\frac{\lambda^{2}}{(4 \pi)^{2}}\left[\log \frac{\lambda \Phi^{2}}{\kappa^{2}}-\frac{3}{2}\right] \\
& -\frac{2 \xi g^{2} \lambda}{(4 \pi)^{2}}\left[\log \frac{\lambda \Phi^{2}}{\kappa^{2}}-1\right]+\frac{\left(\xi g^{2}\right)^{2}}{(4 \pi)^{2}}\left[\log \frac{\xi g^{2}}{\lambda}+\frac{1}{2}\right]
\end{aligned}
$$


The $k_{+}^{2}$ term carries the piece associated with the Goldstone boson in Landau gauge. The $\xi$-dependent terms are of course absent in Landau gauge.

The disconcerting presence of a UV pole in the effective potential is related to the renormalization issues discussed previously. The effective potential in eq. (22) is expressed as a function of a field that has had its $Z_{\phi}$ factor removed only. However, perturbative calculations indicated that an additional shift of the field is necessary to make the 1-PI functions (and thus also $V_{\text {eff }}$ ) finite. A shift in the field by the pole piece of $w$ is sufficient to remove the pole and leave a $V_{\text {eff }}$ which is finite.

It should be noted that the effective potential with the pole is perfectly welldefined. A calculation of the extrema of $V_{\text {eff }}[\Phi]$ yields a one-loop vev with a pole, but the values of $V_{\text {eff }}$ at these extrema are finite and gauge-independent. This is to be expected, since the value of $V_{\text {eff }}$ at extrema corresponds to a physical vacuum energy. However, it is not convenient to work with a divergent $V_{\text {eff }}$ for purposes of studying vacuum stability, and we will choose to work with the expression for the effective potential in terms of the shifted field, $\Phi^{\prime}=\Phi-\delta w_{\text {pole }}$. We might also choose to shift the field by the finite piece, so that the extremum of $V_{\text {eff }}^{\prime}\left[\Phi^{\prime}\right]$ is at $\Phi^{\prime}=0$. Such shifts will be unimportant in the $\Phi^{\prime} \gg \mu, u$ limit, however and we ignore them.

\subsection{Renormalization Group Improvement}

To study $V_{\text {eff }}[\Phi]$ for large $\Phi$, we must use the renormalization group improved effective potential to sum up large $\operatorname{logs}$ of the form $\log \frac{\Phi}{\Phi_{i}}$. Using the invariance of $V_{\text {eff }}[\Phi]$ under changes in $\overline{M S}$ renormalization scale $\kappa$ and dimensional analysis, we obtain an equation for the RG-improved $V_{\text {eff }}$ that will be valid at large fields. This has the solution

$V_{\text {eff }}\left(s \Phi_{i}, \hat{g}_{i}, \mu_{i}, \kappa\right)=\exp \left[\int_{0}^{\log s} \frac{4}{\gamma_{\phi}(x)+1} d x\right] V_{\text {eff }}\left(\Phi_{i}, \hat{g}\left(s, \hat{g}_{i}\right), \hat{\xi}\left(s, \hat{g}_{i}, \hat{\xi}_{i}\right), \mu\left(s, \mu_{i}\right), \kappa\right)$

where

$$
\gamma_{\phi}=-\frac{\kappa}{\phi} \frac{d \phi}{d \kappa}=\frac{1}{2} \frac{\kappa}{Z_{\phi}} \frac{d Z_{\phi}}{d \kappa}
$$

Here $s=\frac{\Phi}{\Phi_{i}}, \hat{g}$ represents the set of couplings $y_{t}, \lambda$, and $g$, and $\hat{\xi}$ represents the pair of gauge parameters $\xi$ and $u$.

Note that $\mu^{2}$ and $u$ do not appear in the large-field limit of $V_{\text {eff }}$ (see eq. (34) and (35)). We also observe that the gauge parameter appears in $V_{\text {eff }}$ only in the combination $\xi g^{2}$, at least at one-loop. The dependence on the gauge parameters then reduces to simply the dependence on $\xi g^{2}$, which is $\mathrm{RG}$ invariant. Thus we can write the RG-improved effective potential for large $\Phi$ as

$$
V_{\text {eff }}\left(s \Phi_{i}, \hat{g}_{i}, \xi g^{2}, \kappa\right)=\frac{1}{4} \lambda_{\text {eff }}\left(\Phi_{i}, \hat{g}\left(s, \hat{g}_{i}\right), \xi g^{2}, \kappa\right)\left(\Phi_{i} \zeta(s)\right)^{4}
$$

where

$$
\zeta(s)=\exp \left[\int_{0}^{\log s} \frac{1}{\gamma_{\phi}(x)+1} d x\right]
$$

It has been shown that the $n$-loop effective potential improved by $n+1$ loop RGEs resums the $n^{\text {th }}$-to-leading logs [13, 14]. This paper does not focus on the 
resummation of the logs, and we are content to sum the leading logs only. Indeed, the issues are perhaps clearest in the region in which relatively small field excursions are necessary and the RG improvement is less important, as is discussed in the next section. It is thus sufficient to consider the one-loop effective potential with one-loop $\beta$ and $\gamma$ functions. In this approximation it is consistent to neglect the running of the couplings $g, y_{t}$, and $\lambda$ in $V_{\text {eff }}^{1}$ and use the one-loop running couplings and fields in $V_{\text {eff }}^{0}$. In this approximation $\Delta \lambda$ is independent of $s$ and $V_{\text {eff }}$ is

$$
V_{\text {eff }}\left(s \Phi_{i}, \hat{g}_{i}, \xi g^{2}, \kappa\right)=\frac{1}{4}\left(\lambda\left(s, \hat{g}_{i}\right)+\Delta \lambda\left(\Phi_{i}, \hat{g}_{i}, \xi g^{2}, \kappa\right)\right)\left(\Phi_{i} \zeta(s)\right)^{4}
$$

where $\Delta \lambda$ is defined in eq.(42). We note that if the running $\lambda(s)$ were used in the $V_{\text {eff }}^{1}$ the effective potential becomes complex for $\lambda(s)<0$.

The $\overline{M S}$ RG equations for the running couplings $g^{2}\left(s, g_{i}^{2}\right)$ and $y_{t}^{2}\left(s, \hat{g}_{i}^{2}\right)$ can be solved analytically:

$$
g^{2}\left(s, g_{i}^{2}\right)=\frac{1}{\frac{1}{g_{i}^{2}}-c_{2} \log s}
$$

where $\beta_{g^{2}}=c_{2} g^{4}$ and

$$
y_{t}^{2}\left(s, \hat{g}_{i}^{2}\right)=\left[\left(\frac{g^{2}(s)}{g_{i}^{2}}\right)^{\frac{c_{4}}{c_{2}}}\left(\frac{1}{y_{i}^{2}}-\frac{c_{3}}{c_{2}+c_{4}} \frac{1}{g_{i}^{2}}\right)+\frac{c_{3}}{c_{2}+c_{4}} \frac{1}{g^{2}(s)}\right]^{-1}
$$

where $\beta_{y_{t}^{2}}=c_{3} y_{t}^{4}-c_{4} y_{t}^{2} g^{2} . y_{t i}^{2}$ and $g_{i}^{2}$ are the initial $(s=1)$ values of the couplings. For $N_{f}=1, c_{2}=\frac{10}{3} \frac{1}{(4 \pi)^{2}}, c_{3}=\frac{11}{(4 \pi)^{2}}$, and $c_{4}=\frac{6}{(4 \pi)^{2}}$. Since this is a $U(1)$ theory and so not asymptotically free, the gauge coupling exhibits a Landau pole at $s=\exp \left[\frac{1}{g_{i}^{2} c_{2}}\right]$. $y_{t}^{2}\left(s, \hat{g}_{i}^{2}\right)$ also exhibits a singularity at

$$
s=\exp \left[\frac{1}{g_{i}^{2} c_{2}}\right] \exp \left[-\frac{1}{c_{2}}\left\{\left(g_{i}^{2}\right)^{-\frac{c_{4}}{c_{2}+c_{4}}}\left(\frac{1}{g_{i}^{2}}-\frac{c_{2}+c_{4}}{c_{3}} \frac{1}{y_{t i}^{2}}\right)^{\frac{c_{2}}{c_{2}+c_{4}}}\right\}\right] .
$$

However, this is not relevant to our analysis as long as the singularities are far beyond the scale at which the electroweak vacuum becomes unstable, and can be arranged without difficulty (for the initial parameters we choose here, the Landau pole of $g^{2}(s)$ is at $s=10^{137}$ and the pole in $y_{t}^{2}(s)$ is at $\left.s=5 \times 10^{13}\right)$. The RG equation for $\lambda\left(s, \hat{g}_{i}^{2}\right)$ must be solved numerically.

\section{The $\overline{M S}$ 'New Physics' Scale and Lower Bounds on the Higgs Mass from Vacuum Stability}

As in the Standard Model, $\beta_{\lambda}$ of our model contains a term due to fermion loops which tends to drive $\lambda(s)$ smaller for increasing $s$. For large $y_{t}^{2}$ (i.e. for a heavy fermion), this term may dominate $\beta_{\lambda}$. At some critical field value of $s, \lambda(s)$ will become negative, and if this occurs for large $s$ the effective potential will quickly 


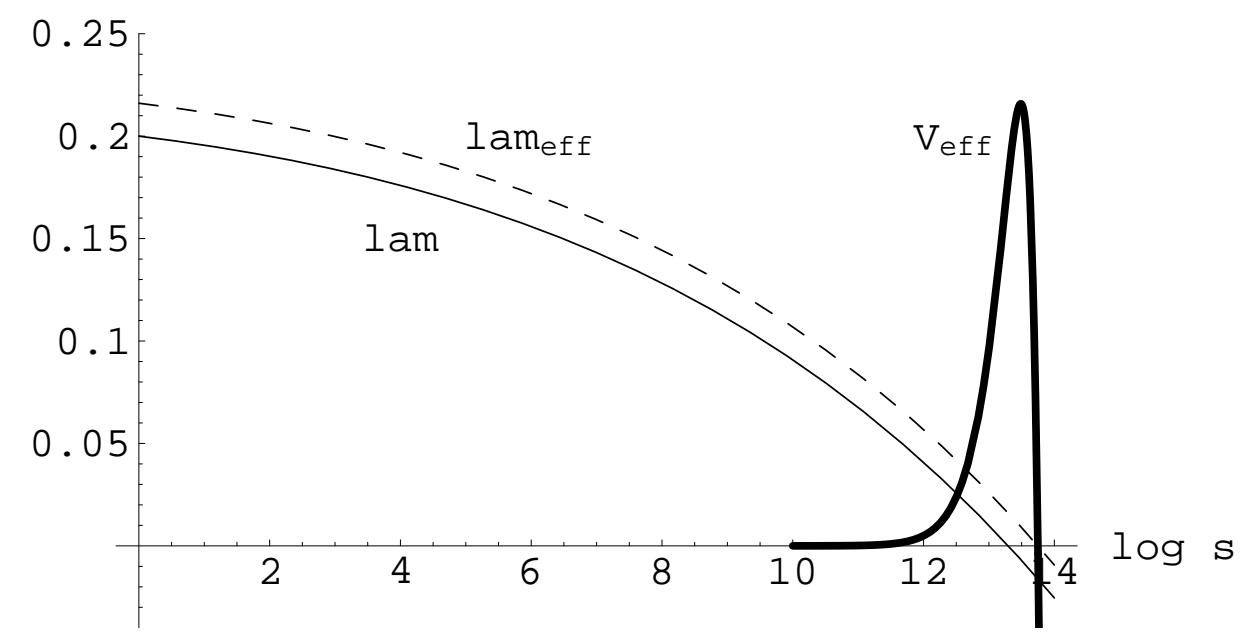

Figure 2: $\lambda_{\text {eff }}(s)$ (dashed line), $\lambda(s)$ (solid line), and $V_{\text {eff }}(s)$ (bold line) vs. $\log s$. $g_{i}^{2}=0.15, y_{t i}^{2}=0.5, \lambda_{i}=0.2, \xi g^{2}=10$. $V_{\text {eff }}$ has been scaled down to fit the plot.

become much lower than the electroweak minimum. If our theory were still complete at this scale it would imply that the electroweak minimum is not global minimum of the theory, contrary to our initial assumption. If we insist that the electroweak vacuum be absolutely stable, we are led to conclude that the theory is incomplete at this scale. Contributions from new physics must be significant at this energy scale and either 'rescue' the effective potential or ruin the entire approach.

Previous studies have considered different criteria for instability of the electroweak vacuum and corresponding specifications of the instability scale. Several papers 15, 16, 17 have taken $\lambda\left(s_{\max }\right)=0$ as specifying the vacuum instability scale, the point at which the RG-improved tree-level effective potential becomes negative. Recently Casas, Espinosa, and Quiros [10, 11, 12 have included one-loop corrections to the Standard Model effective potential (as well as two-loop $\beta$ functions), primarily in an attempt to reduce the renormalization scale $(\kappa)$ dependence of the bounds. They consider the condition $\lambda_{\text {eff }}\left(s_{\max }\right)=0$, where $V_{\text {eff }}\left(s, \Phi_{i}\right) \approx \frac{1}{4} \lambda_{\text {eff }}(s)\left(\Phi_{i} \zeta(s)\right)^{4}$, and observe that this gives a bound on the Higgs mass significantly different from (and presumably better than) that from $\lambda\left(s_{\max }\right)=0$, at least at low cutoff scales. The distinction is illustrated in Fig. 2. There $\lambda_{\text {eff }}\left(s, \xi g^{2}, \hat{g}_{i}\right), \lambda\left(s, \hat{g}_{i}\right)$, and $V_{\text {eff }}$ are plotted

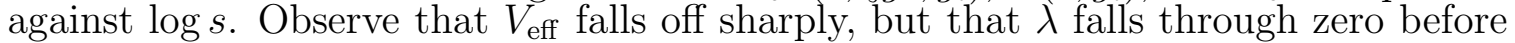
$\lambda_{\text {eff }}$ and $V_{\text {eff }}$ do.

The distinction between the conditions $\lambda(s)=0$ and $\lambda_{\text {eff }}(s)=0$ is equivalent to the distinction between the conditions $V_{\text {eff }}^{0}(s)=0$ and $V_{\text {eff }}^{0}(s)+V_{\text {eff }}^{1}(s)=0 . \lambda(s)$ and $V_{\text {eff }}^{0}$ are gauge independent, but the expression for $\lambda_{\text {eff }}(s)$ in eqs. (36) and (37) contains explicit dependence on the gauge parameter $\xi$ (the dependence on the gauge parameter $u$ has dropped out in this approximation). Thus whether the condition $\lambda_{\text {eff }}(s)=0$ at some assigned $s_{\max }$ or the determination of the instability scale $s_{\max }$ at which $\lambda_{\text {eff }}(s)$ goes to zero is used as the vacuum instability criterion, the result will have explicit gauge $(\xi)$ dependence if one goes beyond the RG improvement of the 
tree-level $V_{\text {eff }}$.

To obtain a lower bound on the Higgs pole mass from one of these stability conditions requires several steps. As input data one needs the values of $g_{i}$ and $y_{t i}$ at some initial low renormalization scale $\kappa_{i}$. In Standard Model studies these would come from global fits to electroweak data (with some error estimate) and introduce no gauge dependence. Then, using the one-loop approximation to the $\beta$ functions one has the RG solutions for running $g$ and $y_{t}$ at any scale (for which they remain perturbatively small). For convenience one may take $\kappa_{i}$ equal to $\Phi_{i}$, the arbitrary electroweak scale at which one specifies the approximate effective potential. One then integrates the RGE for $\lambda(s)$ starting at $s=1\left(\Phi=\Phi_{i}\right)$, with some initial guess for $\lambda(s=1)$. The initial guess is then adjusted until the resulting $\lambda\left(s_{\max }\right)$ at the designated cutoff, $\Phi_{\max }=s_{\max } \Phi_{i}$, satisfies the high-scale boundary condition, $\lambda\left(s_{\max }\right)=-\Delta \lambda$. It is at this stage that the gauge dependence enters, through the explicit $\xi$ dependence of $\Delta \lambda$. The resulting critical $\lambda(s=1)$ becomes the (gauge dependent) lower bound on $\lambda\left(\kappa_{i}\right)(\lambda(\kappa)$ is gauge independent; the bound is gauge dependent).

Alternately, one could start at the high scale $s_{\max }$ with the input $g\left(s_{\max }\right)$ and $y_{t}\left(s_{\max }\right)$ by running the original low scale inputs up to the high scale and by imposing the gauge dependent boundary condition on $\lambda(s), \lambda\left(s_{\max }\right)=-\Delta \lambda$. The RGE for $\lambda(s)$ is then run back down to $s=1$. The result is the same-a gauge dependent lower bound on $\lambda\left(\kappa_{i}\right)$.

There are still some steps to get from a lower bound on $\lambda\left(\kappa_{i}\right)$ to a bound on the Higgs pole mass, but none of them introduce and new (possibly compensating) gauge dependence (they do substantially reduce the dependence on the arbitrarily-chosen initial renormalization scale $\kappa_{i}$.) One requires a numerical value for $v\left(=\sqrt{\frac{-\mu^{2}}{\lambda}}\right)$ the gauge-independent tree-level vev. In the Standard Model, $v^{2}=\frac{1}{\sqrt{2} G_{F}}$ (up to calculated electroweak perturbative corrections). The other ingredient is the relation between the Higgs pole mass $m_{h}^{*}$ and the Higgs $\overline{M S}$ mass $m_{h}^{2}=2 \lambda v^{2}$. This is given by the zero of the inverse propagator at $p^{2}=m_{h}^{* 2}$. Offshell, the inverse propagator is explicitly $\xi$ dependent, and it is a nontrivial check of the calculation that all $\xi$ dependence cancels out onshell. [Note that by its definition as the tree level vev and the renormalization conditions chosen, the relation $v^{2}=\frac{m_{h}^{2}}{2 \lambda}$ gets no perturbative correction].

$$
\begin{aligned}
m_{h}^{* 2}= & m_{h}^{2}+\frac{\lambda m_{h}^{2}}{(4 \pi)^{2}}\left[3 \log \frac{m_{h}^{2}}{\kappa^{2}}+\log \frac{M^{2}}{\kappa^{2}}-12+3 \sqrt{3} \pi+I\left(\frac{m_{h}^{2}}{M^{2}}\right)\right] \\
& +\frac{g^{2} M^{2}}{(4 \pi)^{2}}\left[6+6 I\left(\frac{m_{h}^{2}}{M^{2}}\right)\right]+\frac{g^{2} m_{h}^{2}}{(4 \pi)^{2}}\left[-3 \log \frac{M^{2}}{\kappa^{2}}+1-2 I\left(\frac{m_{h}^{2}}{M^{2}}\right)\right] \\
& +N_{f} \frac{y_{t}^{2} m_{h}^{2}}{(4 \pi)^{2}}\left[2 \log \frac{m_{t}^{2}}{\kappa^{2}}+2 I\left(\frac{m_{t}^{2}}{m_{h}^{2}}\right)\right]-8 N_{f} \frac{y_{t}^{2} m_{t}^{2}}{(4 \pi)^{2}}\left[I\left(\frac{m_{t}^{2}}{m_{h}^{2}}\right)+1\right]
\end{aligned}
$$

where

$$
I(r)=\int_{0}^{1} \log [1-r \alpha(1-\alpha)] d \alpha
$$

In the one-loop corrections to the relation between $m_{h}^{2}$ and $m_{h}^{* 2}$ no distinction is made between $m_{h}^{2}$ and $m_{h}^{* 2}$. Thus, the gauge dependence of the lower bound on $\lambda\left(\kappa_{i}\right)$ 


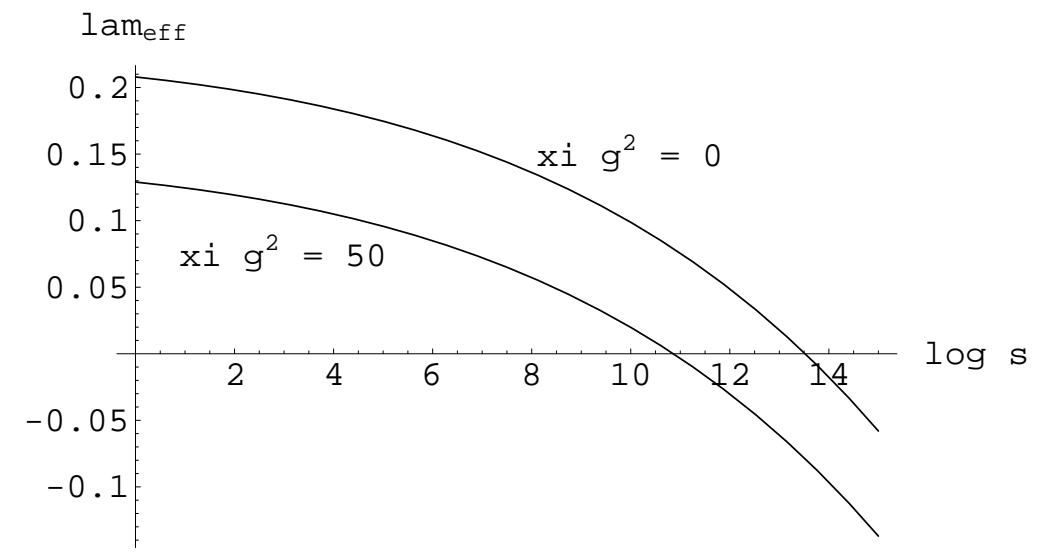

Figure 3: $\lambda_{\text {eff }}\left(s, \xi g^{2}, \hat{g}_{i}\right)$ vs. $\log s$ for $\xi g^{2}=0$ (upper curve) and $\xi g^{2}=50$ (lower curve). $g_{i}^{2}=0.15, y_{t i}^{2}=0.5, \lambda_{i}=0.2$

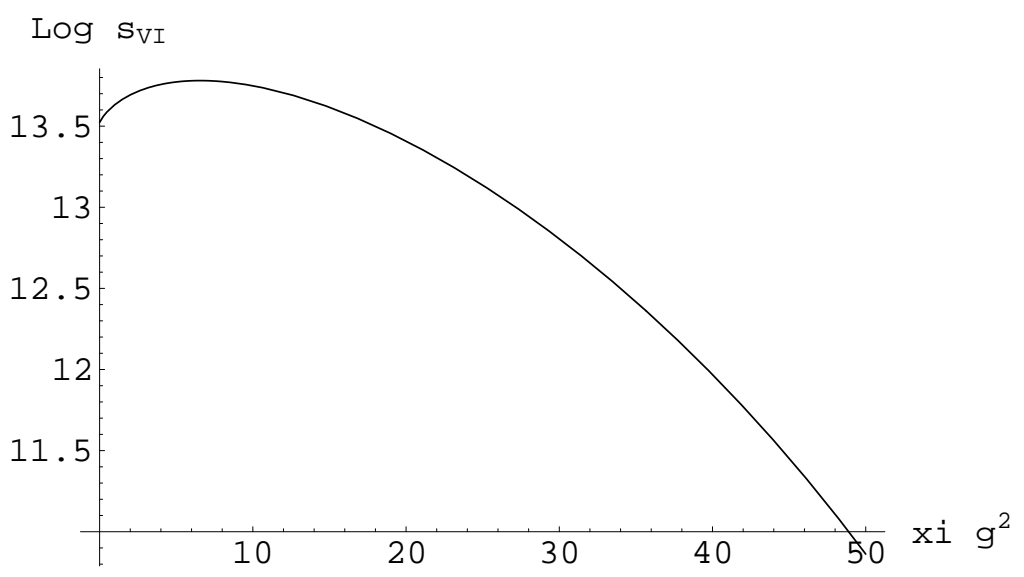

Figure 4: $\log$ of vacuum instability scale vs. $\xi g^{2}$ for $g_{i}^{2}=0.15, y_{t i}^{2}=0.5, \lambda_{i}=0.2$. 


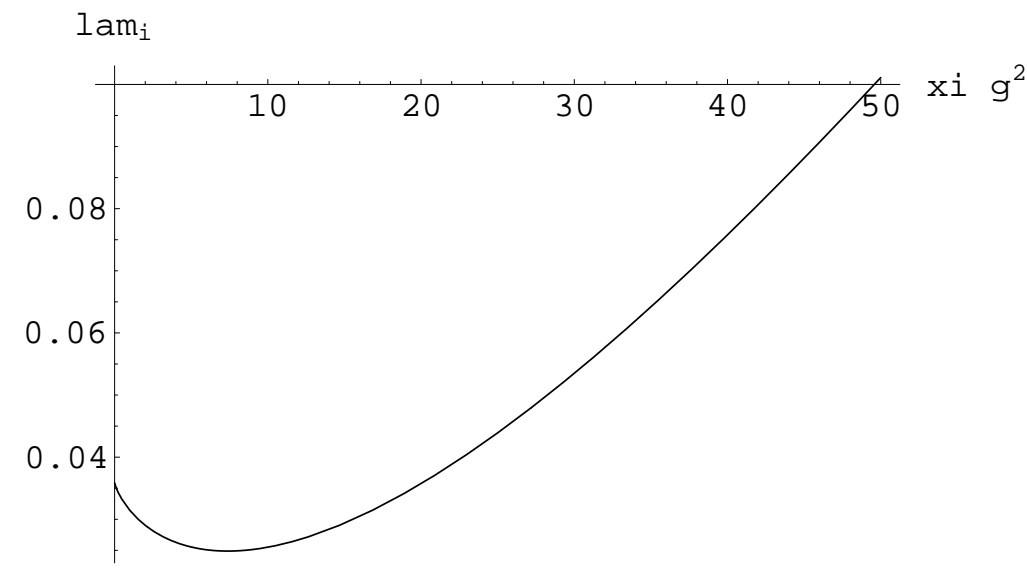

Figure 5: $\lambda_{i}$ vs. $\xi g^{2}$ for $g_{i}^{2}=0.15, y_{t i}^{2}=0.5$, assuming a vacuum instability at $\log s_{V I}=3.7$ and $\lambda_{\text {eff }}\left(s_{V I}\right)=0$.

obtained from the stability condition propagates into the lower bound on the Higgs mass.

To show that the gauge dependence can be made numerically significant, in Fig. 3

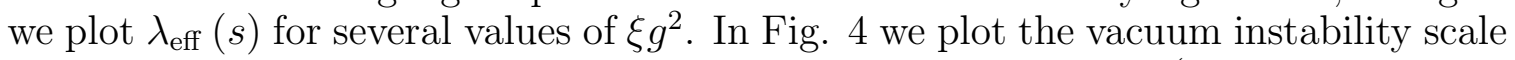
corresponding to a particular lower bound on the Higgs pole mass (which corresponds to some particular $\lambda_{i}$ ) as a function of $\xi g^{2}$. Following the 'top down' approach in Fig. 5, we plot $\lambda_{i}$ as a function of $\xi g^{2}$, assuming that a vacuum instability occurs at $\log s_{V I}=3.7$.

We note also that $V_{\text {eff }}$ contains additional gauge dependence in the scale factor $\zeta(s)$. Since this is an overall exponential multiplicative factor, however, it does not change the point at which $V_{\text {eff }}=0$.

The explicit effects of the $\xi$-dependent terms on the "new physics" scale are easy to see in the region of parameters space in which $\lambda(s) \approx \lambda_{i}+\beta_{\lambda} \log s$ is a good approximation. Solving the equation associated with the turnover of $V_{\text {eff }}^{0}$

$$
\lambda\left(s_{\lambda=0}\right) \approx \lambda_{i}+\beta_{\lambda} \log s_{\lambda=0}=0
$$

gives

$$
s_{\lambda=0}=\exp \left[-\frac{\lambda_{i}}{\beta_{\lambda}}\right]
$$

Considering instead the condition associated with the one-loop effective potential

$$
\lambda_{\text {eff }}\left(s_{\lambda_{\text {eff }}=0}\right)=\lambda(s)+\Delta \lambda \approx \lambda_{i}+\beta_{\lambda} \log s_{\lambda_{\text {eff }}=0}+\Delta \lambda=0
$$

gives

$$
s_{\lambda_{\text {eff }}=0}=s_{\lambda=0} \exp \left[\frac{-\Delta \lambda}{\beta_{\lambda}}\right]
$$

Separating $\Delta \lambda$ into $\xi$-dependent and $\xi$-independent terms

$$
\Delta \lambda=\Delta \lambda_{\xi=0}+\delta \lambda_{\xi g^{2}}
$$


yields

$$
s_{\lambda_{\text {eff }}=0}=s_{\xi=0} \exp \left[\frac{-\delta \lambda_{\xi g^{2}}}{\beta_{\lambda}}\right]
$$

That is, the new physics scale can be expressed in terms of the Landau gauge vacuum instability scale times some gauge-dependent piece which is arbitrary. Thus, we see explicitly that a vacuum instability scale defined in this manner is necessarily gauge dependent.

More generically, we might propose a vacuum instability scale $\omega$ for a gauge choice $\xi$ as the value of the $\Phi$ at which $V_{\text {eff }}[\Phi]$ achieves some value $c$ (c might be zero, as discussed above, or the value of $V_{\text {eff }}$ at one of its extrema, or some other numerical value not dependent on $\xi$ ).

$$
\left[V_{\text {eff }}[\Phi, \xi]\right]_{\Phi=\omega(\xi, c)}=c
$$

Any of these possible definitions correspond to different choices of $c$, but for any $c$ the solution to eq. (59) $\omega(\xi, c)$ will be a function of $\xi$.

\section{Alternative Approaches to the Calculation of Lower Mass Bounds on the Higgs}

In view of these problems of gauge dependence of the effective potential approach, we might consider other approaches which do not make use of the effective potential and are manifestly gauge independent. One such approach has already been proposed by one of the authors [18]. That is to just directly solve the (one-loop, coupled) RGEs for the ratio of the (gauge independent) running $\overline{M S}$ Higgs and top quark masses squared. Requiring that this remain positive up to some cutoff 't Hooft scale, $\kappa_{\max }$, again provides a lower bound on the electroweak scale initial value. One can include the contributions of the gauge couplings with no gauge problems, since the $\beta$ functions for the gauge couplings are gauge independent. A problem with this approach is that a negative running $\overline{M S}$ mass-squared only implies the breakdown of $\overline{M S}$ perturbation theory, not necessarily a disaster of the magnitude of the instability of the vacuum state in which we live. Another problem (shared with the minimal, gauge independent, effective potential approach) is that the connection between the cutoff 't Hooft scale, $\kappa_{\max }$ and the masses of the "new physics" particles is not clear. A simple model in which they are quite different has been give by Hung and Sher [19].

Another possibility is to define a gauge invariant effective potential as the Legendre transform of a source term coupled to a gauge invariant composite operator [20]. While this effective potential is gauge invariant, it is not yet determined whether the problem is computationally tractable.

The problem can also be formulated on a lattice [21]. The Wilson action for the gauge fields is gauge invariant and no gauge fixing term is required. A series of simulations would be run with successively smaller values of the input bare $\lambda_{0}$ and one would look for a nonzero limit for the output ratio of the Higgs to top masses. There will again be the problem of relating the (lattice) cutoff to a scale of new physics. 


\section{Conclusions}

In general it is difficult to extract physical information from the effective potential of a gauge theory, and one might reasonably be skeptical about the accepting the results of an effective potential calculation as physical without some concrete demonstration to that effect. One may argue that physical quantities are independent of gauge, and thus one is free to simply use a convenient gauge. This is certainly true as long as the quantities being calculated are indeed physical quantities in the field theory. However a bound on the Higgs pole mass is not obviously a physical quantity, and the fact that this quantity is gauge-dependent by explicit calculation tells us that in fact it is unphysical, at least as it arises in the field theory. In this case, the expressions for the pole masses of the particles may indeed be expressed in terms of the renormalized couplings and mass parameter and have no explicit gauge dependence order by order in perturbation theory. The RG equations for the couplings are also gauge independent. But the point at which the effective potential attains some particular value is not, and using that information to obtain numerical values of the Higgs pole mass will inevitably introduce gauge dependence into the number. The tree-level $V_{\text {eff }}$ does not suffer from gauge dependence, nor will estimates of the Higgs mass based upon it. However, attempting to incorporate the one-loop $V_{\text {eff }}$ to improve these estimate inescapably introduces uncontrollable gauge dependence even as it reduces the renormalization scale dependence. Hence, no improvement of the tree-level result will be possible using the effective potential with current definitions of the vacuum instability scale. Presumably the issues raised in this context are also applicable to

other problems in which one assigns physical meaning to features of the $\overline{M S}$ effective potential of a model containing a gauge sector (such as the location of minima).

\section{Acknowledgments}

We would like to thank Dan Boyanovsky, Tony Duncan, and Marc Sher for useful conversations.

\section{Appendix A: $\overline{M S}$ renormalization}

\section{$\overline{M S}$ counterterms}

$$
\begin{aligned}
\delta Z_{\phi} & =\left[2 N_{f} y_{t}^{2}-3 g^{2}+\xi g^{2}\right]\left[\frac{-\Delta_{\epsilon}}{(4 \pi)^{2}}\right] \\
\delta Z_{\lambda} & =\left[-10 \lambda+6 g^{2}-3 \frac{g^{4}}{\lambda}-4 N_{f} y_{t}^{2}+4 N_{f} \frac{y_{t}^{4}}{\lambda}\right]\left[\frac{-\Delta_{\epsilon}}{(4 \pi)^{2}}\right] \\
\delta Z_{y} & =\left[-y_{t}^{2}\left(\frac{7}{4}+N_{f}\right)+\frac{3}{2} g^{2}\right]\left[\frac{-\Delta_{\epsilon}}{(4 \pi)^{2}}\right] \\
\delta Z_{\mu^{2}} & =\left[3 g^{2}-4 \lambda-2 N_{f} y_{t}^{2}\right]\left[\frac{-\Delta_{\epsilon}}{(4 \pi)^{2}}\right] \\
\delta Z_{g} & =-\left[\frac{g^{2}}{6}\left(4 N_{f}+1\right)\right]\left[\frac{-\Delta_{\epsilon}}{(4 \pi)^{2}}\right]
\end{aligned}
$$




$$
\begin{aligned}
\delta Z_{B} & =\left[\frac{g^{2}}{3}\left(4 N_{f}+1\right)\right]\left[\frac{-\Delta_{\epsilon}}{(4 \pi)^{2}}\right] \\
\delta Z_{L} & =\left[\xi g^{2}+\frac{y_{t}^{2}}{2}\right]\left[\frac{-\Delta_{\epsilon}}{(4 \pi)^{2}}\right] \\
\delta Z_{t} & =y_{t}^{2}\left[\frac{-\Delta_{\epsilon}}{(4 \pi)^{2}}\right] \\
\delta Z_{c} & =0 \\
\delta Z_{\xi} & =\delta Z_{B} \\
\delta Z_{u} & =-\delta Z_{\phi}
\end{aligned}
$$

\section{$\beta$ functions}

The relevant one-loop $\overline{M S} \beta$ and $\gamma$ functions for the theory are:

$$
\begin{aligned}
\beta_{\lambda} & =\frac{1}{16 \pi^{2}}\left(20 \lambda^{2}-12 \lambda g^{2}+6 g^{4}-8 N_{f} y_{t}^{4}+8 N_{f} \lambda y_{t}^{2}\right) \\
\beta_{g} & =\frac{1}{16 \pi^{2}} g^{3}\left(\frac{4 N_{f}+1}{3}\right) \\
\beta_{y_{t}} & =\frac{1}{16 \pi^{2}}\left(\left(\frac{7}{4}+N_{f}\right) 2 y_{t}^{3}-3 g^{2} y_{t}^{2}\right) \\
\gamma_{\phi} & =\frac{1}{16 \pi^{2}}\left(2 N_{f} y_{t}^{2}-g^{2}(3-\xi)\right)
\end{aligned}
$$

where $N_{f}$ is the number of copies of fermion doublet (all assumed to have the same couplings). 


\section{References}

[1] Marc Sher, Phys. Rep. 89273 (1979).

[2] L. Dolan, R. Jackiw, Phys. Rev. D9 2904 (1974).

[3] K. Fujikawa, B.W. Lee, A.I. Sandia, Phys. Rev. D6 2923 (1972).

[4] Benjamin W. Lee, Phys. Rev. D9 933 (1974).

[5] Thomas Appelquist, J. Carazzone, T. Goldman, and H.R. Quinn, Phys. Rev. D8 1747 (1973).

[6] Reijiro Fukada, Taichiro Kugo, Phys. Rev. D13 3469 (1976).

[7] Erick J. Weinberg, Aiqun Wu, Phys. Rev. D36 2474 (1987).

[8] S.Y. Lee, Alain M. Sciaccaluga, Nucl. Phys. B96 435 (1975).

[9] I.J.R. Aitchison, C.M. Fraser, Ann. Phys. 1561 (1984).

[10] J.A. Casas, J.R. Espinosa, M. Quiros, Phys. Lett. B342 171 (1995).

[11] J.A. Casas, J.R. Espinosa, M. Quiros, Nucl. Phys. B436 3 (1995).

[12] J.A. Casas, J.R. Espinosa, M. Quiros, Phys. Lett. B382 374 (1996).

[13] Masako Bando, Taichiro Kugo, Nobuhiro Maekawa, Hiroaki Nakano, Phys. Lett. B301 83 (1993).

[14] Boris Kastening, Phys. Lett. B283 287 (1992).

[15] G. Altarelli, G. Isidori Phys. Lett. B337 141 (1994).

[16] Marc Sher, Phys. Lett. B317 59 (1993), Addendum Phys. Lett. B331 448 (1994).

[17] C. Ford, D.R.T. Jones, P.W. Stephenson, M.B. Einhorn, Nucl. Phys. B395 17 (1993).

[18] R.S. Willey, Phys. Lett. B377 255 (1996), (E) B381 498 (1996), hep-ph/9607283.

[19] P.Q. Hung, Marc Sher, Phys. Lett. B74 138 (1996).

[20] T. Banks, S. Raby, Phys. Rev. D14 2182 (1976).

[21] L. Lin, I. Montvay, H. Wittig, G. Munster, Nucl. Phys. B355 511 (1991). 